\title{
FREE ADEQUATE SEMIGROUPS
}

\author{
MARK KAMBITES \\ (Received 17 July 2010; accepted 19 February 2011) \\ Communicated by M. G. Jackson
}

\begin{abstract}
We give an explicit description of the free objects in the quasivariety of adequate semigroups, as sets of labelled directed trees under a natural combinatorial multiplication. The morphisms of the free adequate semigroup onto the free ample semigroup and into the free inverse semigroup are realised by a combinatorial 'folding' operation which transforms our trees into Munn trees. We use these results to show that free adequate semigroups and monoids are $\mathcal{J}$-trivial and never finitely generated as semigroups, and that those which are finitely generated as $(2,1,1)$-algebras have decidable word problem.
\end{abstract}

2010 Mathematics subject classification: primary 20M10; secondary 08A10, 08A50.

Keywords and phrases: adequate semigroup, free object, word problem, Munn tree.

\section{Introduction}

The structural theory of semigroups has traditionally been largely concerned with semigroups which admit local inverses with respect to nonidentity idempotents; chief among these are the regular and inverse semigroups. In the late 1970s, it was observed by Fountain $[6,7]$ that many of the desirable properties of regular and inverse semigroups stem not directly from the existence of local inverses, but rather from the consequent fact that the cancellation properties of elements in such a semigroup are reflected in cancellation properties of the idempotents. This observation opened up effective methods of study for much wider classes of semigroups, the development of which forms the basis of the York school of semigroup theory.

Amongst the classes of semigroups introduced by the York school, the oldest and perhaps most natural is the class of adequate semigroups. Adequate semigroups generalise inverse semigroups in something akin to the way that cancellative monoids generalise groups; indeed the single-idempotent adequate monoids are exactly the cancellative monoids, in the same way that the single-idempotent inverse monoids are exactly the groups. They relate to abundant semigroups [7] in the same way

Supported by an RCUK Academic Fellowship.

(C) 2012 Australian Mathematical Publishing Association Inc. 1446-7887/2012 \$16.00 
that inverse semigroups relate to regular semigroups. Adequate semigroups are most naturally viewed as algebras (in the sense of universal algebra) of signature $(2,1,1)$, where the usual multiplication is augmented with unary operations $*$ and + which map each element to certain idempotents which share its left and right cancellativity properties (see Section 2 for a precise definition). Within the category of $(2,1,1)$ algebras (although not in the category of semigroups) the adequate semigroups form a quasivariety, and it follows from general principles (see, for example, [4, Proposition VI.4.5]) that there exists a free adequate semigroup for each cardinality of generating set.

When studying any class of algebras, it is extremely helpful to have an explicit combinatorial description of the free objects in the class. Such a description allows one, for example, to understand which identities do and do not hold in the given class, and potentially to express any member of the class as a collection of equivalence classes of elements in a free algebra. In the case of inverse semigroups, a description of the free objects first discovered by Scheiblich [22] was developed by Munn [18] into an elegant geometric representation which has been of immense value in understanding inverse semigroups. Subsequently there have appeared a number of alternative proofs of Munn's result and different representations for the free inverse semigroup [13, 1921]. Variants of Munn's approach have since been used to describe the free objects in a number of more general classes of semigroups [8-10] and also in the closely related setting of Cockett-Lack restriction categories [2]. All of these techniques rely on certain identities satisfied by the classes of semigroups (or categories) in question. These identities permit the rewriting of expressions to move idempotents to one side, and hence allow the systematic decomposition of each element as a product of an idempotent part and an element of a free subsemigroup. In a free adequate semigroup, by contrast, a typical element cannot be written as such a product, so there is no hope of directly applying Munn's technique, and hitherto no explicit description of the free adequate semigroup has been found.

The main aim of this paper is to give an explicit geometric representation of the free adequate semigroup on a given set, as a collection of edge-labelled directed trees under a natural multiplication operation. This result is inspired by Munn's celebrated characterisation of free inverse semigroups as subtrees of the Cayley graph of the free group [18]; indeed the natural map from a free adequate semigroup to the free inverse semigroup admits a natural interpretation as a 'folding' map of our trees onto Munn trees. Partly for this reason, we believe that our representation is the natural analogue of Munn's for adequate semigroups, and is likely to prove correspondingly useful in the study of adequate semigroups.

As examples of how our main theorems can be applied, we show that every free adequate semigroup or monoid is $\mathcal{J}$-trivial, while nontrivial examples are never finitely generated as semigroups. Our representation also gives rise to a nondeterministic polynomial time decision algorithm for the word problem in these semigroups; the exact computational complexity of the word problem remains for now unclear, and deserves further study. 
We show elsewhere [16] that our approach also leads to a description of the free objects in the categories of left and right adequate semigroups (roughly speaking, those semigroups which satisfy the conditions defining adequate semigroups on one side only). An alternative approach to free left and right adequate semigroups appears in recent work of Branco et al. [1, 11].

In addition to this introduction, this paper comprises six sections. In Section 2 we briefly recall the definition of adequate semigroups, and summarise some of their elementary properties on which we later rely. Sections 3 and 4 introduce respectively the basic combinatorial objects from which our representation is constructed, and the operations we shall use for combining them, as well as proving some foundational results concerning them. Section 5 is devoted to the proof that the resulting algebraic structures are free objects in the quasivariety of adequate semigroups. Section 6 contains remarks on our characterisation and its relationship with other work, while Section 7 shows how it can be applied to establish some basic algebraic properties of the free adequate semigroups and monoids.

\section{Preliminaries}

In this section we briefly recall some definitions, notation and terminology relating to adequate semigroups. For a more comprehensive and detailed introduction, see [6].

Recall that if $S$ is a semigroup without identity then $S^{1}$ denotes the monoid obtained by adjoining an additional element to $S$ which acts as a multiplicative identity element; if $S$ is already a monoid then we define $S^{1}=S$. On any semigroup $S$, an equivalence relation $\mathcal{L}^{*}$ is defined by $a \mathcal{L}^{*} b$ if and only if

$$
a x=a y \Longleftrightarrow b x=b y
$$

holds for every $x, y \in S^{1}$. Dually, an equivalence relation $\mathcal{R}^{*}$ is defined by $a \mathcal{R}^{*} b$ if and only if

$$
x a=y a \Longleftrightarrow x b=y b
$$

holds for every $x, y \in S^{1}$.

A semigroup is called left abundant (right abundant) if every $\mathcal{R}^{*}$-class (respectively, every $\mathcal{L}^{*}$-class) contains an idempotent. A semigroup is abundant if it is both left abundant and right abundant. If an abundant (left abundant, right abundant) semigroup has the additional property that the idempotents commute, then the semigroup is called adequate (left adequate, right adequate). It is easily seen that, in a left (right) adequate semigroup, each $\mathcal{R}^{*}$-class $\left(\mathcal{L}^{*}\right.$-class) must contain a unique idempotent. We denote by $x^{+}$(respectively, $x^{*}$ ) the unique idempotent in the $\mathcal{R}^{*}$-class (respectively, $\mathcal{L}^{*}$-class) of an element $x$; this idempotent acts as a left (right) identity for $x$. The unary operations $x \mapsto x^{+}$and $x \mapsto x^{*}$ are of such critical importance in the theory of adequate (left adequate, right adequate) semigroups that it is usual to consider these semigroups as algebras of signature $(2,1,1)$ (or $(2,1)$ for left adequate and right adequate semigroups) with these operations. In particular, one restricts attention to 
morphisms which preserve the + and/or $*$ operations (and hence coarsen the $\mathcal{R}^{*}$ and $\mathcal{L}^{*}$ relations) as well as the multiplication. These form a proper subclass of the semigroup morphisms between the adequate semigroups, as can be seen by considering, for example, any map from a free monoid (which is cancellative and hence adequate) onto any adequate monoid with more than one idempotent. Similarly, adequate monoids may be viewed as algebras of signature $(2,1,1,0)$, and left or right adequate monoids may be viewed as algebras of signature $(2,1,0)$, with the identity a distinguished constant symbol.

We mention one important subclass of the adequate semigroups. An adequate semigroup $S$ is called ample (also known as type $A$ ) if $a e=(a e)^{+} a$ and $e a=a(e a)^{*}$ for all elements $a \in S$ and idempotents $e \in S$.

We now establish some basic properties of left and right adequate semigroups; these are well known but since the proofs are very short we include them in order to keep this paper self-contained.

Proposition 2.1. Let $S$ be a left adequate (respectively, right adequate) semigroup and let $a, b, e, f \in S$ with $e$ and $f$ idempotent. Then

(i) $e^{+}=e$

(ii) $(a b)^{+}=\left(a b^{+}\right)^{+}$

(iii) $a^{+} a=a$

(iv) $e a^{+}=(e a)^{+}$

(v) $a^{+}(a b)^{+}=(a b)^{+}$

(vi) if ef $=f$ then $(a e)^{+}(a f)^{+}=(a f)^{+}$ (respectively, $\left.e=e^{*}\right)$;

(respectively, $\left.(a b)^{*}=\left(a^{*} b\right)^{*}\right)$;

(respectively, $a a^{*}=a$ );

(respectively, $\left.a^{*} e=(a e)^{*}\right)$;

(respectively, $(a b)^{*} a^{*}=\left(a b^{*}\right)$ );

(respectively, $\left.(e a)^{*}(f a)^{*}=(f a)^{*}\right)$.

PRoof. In each case we prove only the claim for left adequate semigroups, the other being dual.

(i) By definition $e^{+}$is the unique idempotent in the $\mathcal{R}^{*}$-class of $e$, which, since $e$ is idempotent, must be $e$ itself.

(ii) Since $a b \mathcal{R}^{*}(a b)^{+}$we have $x(a b)^{+}=y(a b)^{+}$if and only if $x a b=y a b$. Since $b \mathcal{R}^{*} b^{+}$this is true if and only if $x a b^{+}=y a b^{+}$. And since $a b^{+} \mathcal{R}^{*}\left(a b^{+}\right)^{+}$this is true if and only if $x\left(a b^{+}\right)^{+}=y\left(a b^{+}\right)^{+}$. Thus, $(a b)^{+} \mathcal{R}^{*}\left(a b^{+}\right)^{+}$. But both are idempotent and each $\mathcal{R}^{*}$-class contains a unique idempotent, so we must have $(a b)^{+}=\left(a b^{+}\right)^{+}$.

(iii) Since $a^{+} \mathcal{R}^{*} a$ and $a^{+} a^{+}=a^{+}=1 a^{+}$we have $a^{+} a=1 a=a$.

(iv) Since idempotents commute, $\left(e a^{+}\right)\left(e a^{+}\right)=e e a^{+} a^{+}=e a^{+}$, that is, $e a^{+}$is idempotent. Now using (i) and (ii), $e a^{+}=\left(e a^{+}\right)^{+}=\left(e a^{+}\right)^{+}=(e a)^{+}$.

(v) Using (iv) and (iii), $a^{+}(a b)^{+}=\left(a^{+} a b\right)^{+}=(a b)^{+}$.

(vi) We have $(a e)^{+}(a f)^{+}=(a e)^{+}(a e f)^{+}=(a e f)^{+}=(a f)^{+}$, where the second equality is an application of part (v).

Recall that an object $F$ in a concrete category $C$ is called free on a subset $\Sigma \subseteq F$ if every function from $\Sigma$ to an object $N$ in $C$ extends uniquely to a morphism from $F$ to $N$. The subset $\Sigma$ is called a free generating set for $F$, and its cardinality is the rank of $F$. 
A free object in a given concrete category is uniquely determined up to isomorphism by its rank, so it is usual to speak of the free object of a given rank in a given category.

Within the class of $(2,1,1)$-algebras, the adequate semigroups form a quasivariety, defined by the quasiidentities

$$
A X=A Y \Longleftrightarrow A^{*} X=A^{*} Y
$$

and

$$
X A=Y A \Longleftrightarrow X A^{+}=Y A^{+},
$$

together with the associative law for multiplication, and further identities which ensure that the unary operations are idempotent and have idempotent and commutative images. A corresponding statement applies to the class of adequate monoids. Since every quasivariety contains free objects of every rank (see, for example, [4, Proposition VI.4.5]) it follows that there exist free adequate semigroups and monoids of every rank. The chief aim of the present paper is to give an explicit geometric representation of these. We begin with a proposition, the essence of which is that the distinction between semigroups and monoids is unimportant.

Proposition 2.2. Let $\Sigma$ be an alphabet. The free adequate monoid on $\Sigma$ is isomorphic to the free adequate semigroup on $\Sigma$ with a single adjoined element which is an identity for multiplication and a fixed point for $*$ and + .

Proof. Let $S$ be the free adequate semigroup on $\Sigma$, and let $T=S \cup\{1\}$ be the monoid obtained by adjoining an element 1 which is a multiplicative identity and fixed point for $*$ and + . Then certainly $T$ is a monoid, and it is easily verified from the definitions that $T$ is adequate. Now suppose that $f: \Sigma \rightarrow M$ is a map from $\Sigma$ to an adequate monoid. Since $S$ is free on $\Sigma$ and the monoid $M$ is also an adequate semigroup, $f$ extends uniquely to a $(2,1,1)$-morphism $g: S \rightarrow M$. We define a map $h: T \rightarrow M$ by $h(1)=1$ and $h(x)=g(x)$ for all $x \in S$. Then it is easily verified that $h$ is a $(2,1,1,0)$ morphism from $T$ to $M$ which extends $f$. Moreover, $h$ is the unique morphism with this property, since any other such morphism would restrict to another morphism from $S$ to $M$ extending $f$, contradicting the assumption that $S$ is free. Thus, $T$ is a free adequate monoid on $\Sigma$.

We discuss briefly the relationship of abundant and adequate semigroups to regular and inverse semigroups. Recall that Green's relation $\mathcal{L}$ is defined on any semigroup $S$ by $x \mathcal{L} y$ if and only if $x$ and $y$ generate the same principal left ideal. Similarly, Green's relation $\mathcal{R}$ is defined by $x \mathcal{R} y$ if and only if $x$ and $y$ generate the same principal right ideal. A semigroup is called regular if every $\mathcal{R}$-class and every $\mathcal{L}$-class contains an idempotent; a regular semigroup is called inverse if in addition the idempotents commute. It can be shown [6] that two elements of $S$ are $\mathcal{L}^{*}$-related (respectively, $\mathcal{R}^{*}$-related) if and only if there is an embedding of $S$ into another semigroup in which their images generate the same principal left ideal (respectively, principal right ideal). It follows that $\mathcal{L}^{*}$ and $\mathcal{R}^{*}$ are coarsenings of $\mathcal{L}$ and $\mathcal{R}$, and hence that every regular 

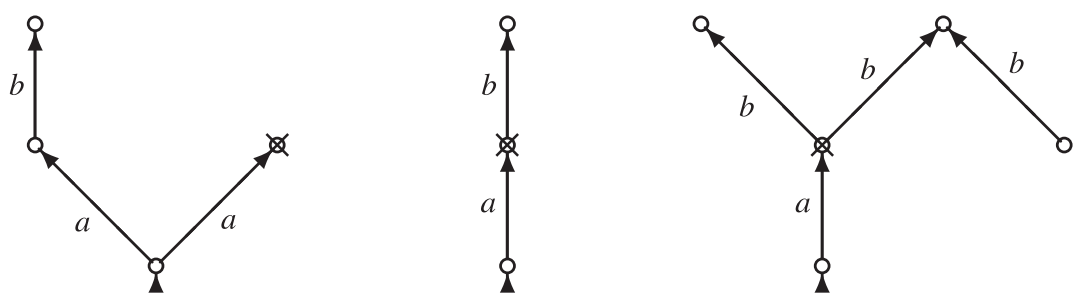

Figure 1. Some examples of $\{a, b\}$-trees.

semigroup is abundant and every inverse semigroup is adequate. In fact, it can be shown that every inverse semigroup is ample.

\section{Trees and pruning}

In this section we introduce the combinatorial objects which will form the elements of our representation of the free adequate semigroup. The main objects of our study are labelled directed trees, by which we mean edge-labelled directed graphs whose underlying undirected graphs are trees. Note that such graphs have the property that there is at most one directed path between any two vertices. If $e$ is an edge in such a tree, we denote by $\alpha(e), \omega(e)$ and $\lambda(e)$ the vertex at which $e$ starts, the vertex at which $e$ ends and the label of $e$, respectively.

Definition 3.1 ( $\Sigma$-trees). Let $\Sigma$ be an alphabet. A $\Sigma$-tree (or just a tree if the alphabet $\Sigma$ is clear) is a directed tree with edges labelled by elements of $\Sigma$, and with two distinguished vertices (the start vertex and the end vertex) such that there is a (possibly empty) directed path from the start vertex to the end vertex.

A tree with only one vertex is called trivial, while a tree with start vertex equal to its end vertex is called idempotent. A tree with a single edge and distinct start and end vertices is called a base tree; we identify each base tree with the label of its edge.

In any tree, the (necessarily unique) directed path from the start vertex to the end vertex is called the trunk of the tree; the vertices of the graph which lie on the trunk (including the start and end vertices) are called trunk vertices and the edges which lie on the trunk are called trunk edges. If $X$ is a tree we write $\theta(X)$ for the set of trunk edges of $X$.

Figure 1 shows three examples of $\Sigma$-trees where $\Sigma=\{a, b\}$. In each case, the start vertex is marked by an arrow-head, and the end vertex by a cross. Notice that a vertex may have multiple edges coming in or going out with the same label, and that in each case there is a directed path (in our examples, a single edge) from the start vertex to the end vertex. Figure 2 shows the trivial tree and the base trees $a$ and $b$.

Definition 3.2 (Subtrees and morphisms). Let $X$ and $Y$ be $\Sigma$-trees. A subtree of $X$ is a subgraph of $X$ with the same start and end vertices, the underlying undirected graph of which is connected. 


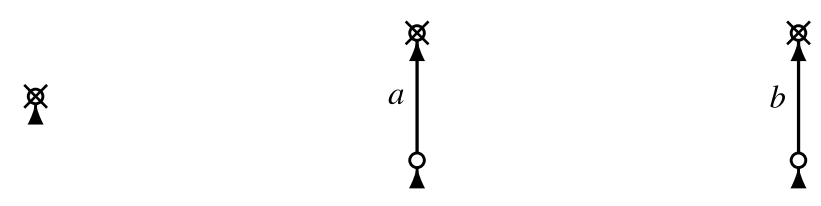

FIGURE 2. The trivial tree, the base tree $a$ and the base tree $b$.

A morphism $\rho: X \rightarrow Y$ of $\Sigma$-trees is a map taking edges to edges and vertices to vertices, such that $\rho(\alpha(e))=\alpha(\rho(e)), \rho(\omega(e))=\omega(\rho(e))$ and $\lambda(e)=\lambda(\rho(e))$ for all edges $e$ in $X$, and which maps the start and end vertex of $X$ to the start and end vertex of $Y$, respectively. An isomorphism is a morphism which is bijective on both edges and vertices.

In our example of Figure 1, there is clearly a unique morphism from the left-hand tree to the middle tree, and a unique morphism taking the right-hand tree to the middle tree.

It is easily shown that morphisms have the expected properties that the composition of two morphisms (where defined) is again a morphism, while the restriction of a morphism to a subtree is also a morphism. It is also easily verified (using the fact that we consider only trees with a directed path from the start to the end vertex) that a morphism necessarily maps the trunk edges of its domain bijectively onto the trunk edges of its image. Note that morphisms map $\Sigma$-trees to $\Sigma$-trees (for the same alphabet $\Sigma$ ), and preserve the labelling of edges.

Definition 3.3. The set of all isomorphism types of $\Sigma$-trees is denoted $U T^{1}(\Sigma)$, while the set of isomorphism types of nontrivial $\Sigma$-trees is denoted $U T(\Sigma)$. The set of isomorphism types of idempotent trees is denoted $U E^{1}(\Sigma)$, while the set of isomorphism types of nontrivial idempotent trees is denoted $U E(\Sigma)$.

Much of the time we shall be formally concerned not with trees themselves but rather with isomorphism types. However, where no confusion is likely, we shall for the sake of conciseness ignore the distinction and implicitly identify trees with their respective isomorphism types.

Definition 3.4 (Retracts). A retraction of a tree $X$ is an idempotent morphism from $X$ to $X$; its image is called a retract of $X$. A tree $X$ is called pruned if it does not admit a nonidentity retraction. The set of all isomorphism types of pruned trees (respectively, nontrivial pruned trees) is denoted $T^{1}(\Sigma)$ (respectively, $T(\Sigma)$ ).

Returning to our examples from Figure 1, neither the left-hand nor the middle tree admits any nonidentity retraction, so these trees are pruned. The right-hand tree admits four nonidentity retractions. Note also that the trivial tree and the base trees, examples of which are shown in Figure 2, do not admit any nonidentity retractions, and so are pruned trees. 
The restriction of a retraction to a subtree containing its image is again a retraction. A composition of retractions is not in general a retraction, but it will be whenever the domain of the second is exactly the image of the first. The following proposition is an instance of a well-known phenomenon, another important example of which is the uniqueness of the core of a finite graph [14, 15]. It can be deduced from very general results about morphisms of finite relational structures (for example, [5, Proposition 1.4.7]) but for completeness we sketch a simple combinatorial proof.

Proposition 3.5 (Confluence of retracts). For each tree $X$ there is a unique (up to isomorphism) pruned tree which is a retract of $X$.

Proof. Clearly, since $X$ is finite it has a retract of minimal size, which must be a pruned tree. Suppose now that $\pi_{1}: X \rightarrow X$ and $\pi_{2}: X \rightarrow X$ are both retractions with pruned images $Y$ and $Z$, respectively. Consider the two compositions $\pi_{1} \pi_{2}$ and $\pi_{2} \pi_{1}$; since they are maps on a finite set we may choose a positive integer $n$ such that $\left(\pi_{1} \pi_{2}\right)^{n}$ and $\left(\pi_{2} \pi_{1}\right)^{n}$ are idempotent morphisms, that is, retractions of $X$. A straightforward argument shows that the restriction of $\left(\pi_{1} \pi_{2}\right)^{n}$ to $Y$ is a retraction of $Y$, which since $Y$ is pruned means it must be the identity map on $Y$. Dually, the restriction of $\left(\pi_{2} \pi_{1}\right)^{n}$ to $Z$ is the identity map on $Z$. It now follows easily that $\pi_{1}$ and $\pi_{2}\left(\pi_{1} \pi_{2}\right)^{n-1}$ restrict to give mutually inverse isomorphisms between $Y$ and $Z$, as required.

Definition 3.6 (Pruning of a tree). Let $X \in U T^{1}(\Sigma)$. Then the pruning of $X$ is the unique (by Proposition 3.5) element of $T^{1}(\Sigma)$ which can be obtained from $X$ by pruning. It is denoted $\bar{X}$.

Considering again our examples from Figure 1, we have already seen that the lefthand and middle trees are pruned, and so each is (or more properly, the isomorphism type of each is) its own pruning. Two of the four retracts of the right-hand tree are pruned; Proposition 3.5 tells us that these must be isomorphic, and indeed they are both isomorphic to the middle tree of Figure 1. Hence, the pruning of the right-hand tree is (the isomorphism type of) the middle tree.

\section{Algebra on trees}

We now define some operations on isomorphism types of trees.

Definition 4.1 (Unpruned operations). We define a product operation, called unpruned multiplication, on $U T^{1}(\Sigma)$ as follows. For $X, Y \in U T^{1}(\Sigma)$, choose representative $\Sigma$-trees $X^{\prime}$ for $X$ and $Y^{\prime}$ for $Y$ such that $X^{\prime} \cap Y^{\prime}=\{v\}$ where $v$ is the end vertex of $X^{\prime}$ and the start vertex of $Y^{\prime}$. Then the unpruned product $X \times Y$ is the isomorphism type of the tree with graph $X^{\prime} \cup Y^{\prime}$ (with the maps $\alpha, \omega$ and $\lambda$ extending the corresponding maps in $X^{\prime}$ and $Y^{\prime}$ ) considered as a $\Sigma$-tree with start vertex the start vertex of $X^{\prime}$ and end vertex the end vertex of $Y^{\prime}$.

We also define two unary operations on $U T^{1}(\Sigma)$, called unpruned $(+)$ and unpruned (*). If $X^{\prime}$ is a representative $\Sigma$-tree for $X \in U T^{1}(\Sigma)$ then $X^{(+)}$is the isomorphism type of 
the idempotent tree with the same underlying graph and start vertex as $X^{\prime}$, but with end vertex the start vertex of $X^{\prime}$. Dually, $X^{(*)}$ is the isomorphism type of the idempotent tree with the same underlying graph and end vertex as $X^{\prime}$, but with start vertex the end vertex of $X^{\prime}$.

The above definition is rendered rather technical by the formal need to work with representatives of isomorphism types, but intuitively the operations are very simple. For example, unpruned multiplication simply means 'gluing together' two trees by identifying the end vertex of one with the start vertex of the other.

Returning to our example trees from Figure 1, and recalling that we identify the letters $a$ and $b$ with the corresponding base trees (as shown in Figure 2), we see that the trees depicted correspond to the unpruned expressions $(a \times b)^{(+)} \times a, a \times b^{(+)}$and $a \times b^{(+)} \times\left(b \times b^{(*)}\right)^{(+)}$, respectively.

The following proposition gives some elementary properties of these operations.

Proposition 4.2. Unpruned multiplication is an associative binary operation on the set $U T^{1}(\Sigma)$ of isomorphism types of $\Sigma$-trees. The isomorphism type of the trivial tree is an identity element for this operation. The set $U T(\Sigma)$ of isomorphism types of nontrivial trees forms a subsemigroup of $U T^{1}(\Sigma)$.

The maps $X \mapsto X^{(+)}$and $X \mapsto X^{(*)}$ are idempotent unary operations on $U T^{1}(\Sigma)$. The subsemigroup generated by the images of these unary operations is commutative.

Proof. It is easily seen that unpruned multiplication is associative, that the trivial tree acts as an identity, and that the product of two nontrivial trees is never trivial. Finally, the images of the $(+)$ and $(*)$ operations are by definition idempotent trees, and it is immediate from the definitions that multiplication of idempotent trees is commutative.

Definition 4.3 (Pruned operations). Let $X$ and $Y$ be isomorphism types of pruned trees. Then we define $X Y=\overline{X \times Y}, X^{*}=\overline{X^{(*)}}$ and $X^{+}=\overline{X^{(+)}}$.

Proposition 4.4. Pruned multiplication is a well-defined binary operation on the set $T^{1}(\Sigma)$ of isomorphism types of pruned trees. The unary operations $*$ and + are welldefined idempotent unary operations on the set $T^{1}(\Sigma)$ of isomorphism types of pruned trees.

Proof. The claims follow easily from Proposition 4.2.

Definition 4.3 and Proposition 3.5 provide the reason why we must formally work with isomorphism types of trees rather than trees themselves. Since choices made during the process of 'pruning' may result in distinct but isomorphic pruned trees, pruned multiplication is a well-defined operation only on isomorphism types, and not on trees themselves. 
We are now ready to prove a basic but important foundational result.

Theorem 4.5. The pruning map

$$
U T^{1}(\Sigma) \rightarrow T^{1}(\Sigma), \quad X \mapsto \bar{X}
$$

is a surjective $(2,1,1,0)$-morphism from the set of isomorphism types of $\Sigma$-trees under unpruned multiplication, unpruned $(*)$ and unpruned $(+)$ with distinguished identity element to the set of isomorphism types of pruned trees under pruned multiplication, * and + with distinguished identity element.

Proof. First notice that every isomorphism type $X \in T^{1}(\Sigma)$ of pruned trees is also an isomorphism type of $\Sigma$-trees and satisfies $\bar{X}=X$; thus, the given map is surjective.

Now let $X$ and $Y$ be unpruned trees. Let $\pi_{X}: X \rightarrow X$ and $\pi_{Y}: Y \rightarrow Y$ be retractions with images $\bar{X}$ and $\bar{Y}$, respectively.

We show first that $\bar{X} \bar{Y}=\overline{X \times Y}$. Notice that since the amalgamated vertex in the unpruned product $X \times Y$ is the end vertex of $X$ and the start vertex of $Y$, it is fixed by both $\pi_{X}$ and $\pi_{Y}$; it follows that there is a (necessarily unique, since every vertex and edge of $X \times Y$ comes from $X$ or $Y$ ) map $\pi: X \times Y \rightarrow X \times Y$ which extends both $\pi_{X}$ and $\pi_{Y}$. Clearly, $\pi$ is a morphism. Since $\pi_{X}$ and $\pi_{Y}$ are idempotent and at least one of them is defined on each vertex and edge of $X \times Y$, we see also that $\pi$ is idempotent, and hence is a retraction. Moreover, it follows immediately from the definition of unpruned multiplication that $\pi(X \times Y)=\bar{X} \times \bar{Y}$. But now, by Proposition 3.5,

$$
\overline{X \times Y}=\overline{\pi(X \times Y)}=\overline{\bar{X} \times \bar{Y}}=\overline{X Y} .
$$

Next we claim that $\bar{X}^{+}=\overline{X^{(+)}}$. First notice that, since $X^{(+)}$has the same underlying labelled directed graph as $X$, the same start vertex, and end vertex the start vertex of $X$, the retraction $\pi_{X}$ of $X$ is also a retraction of $X^{(+)}$. Clearly, its image is the tree $\bar{X}^{(+)}$. Hence, by Proposition 3.5 again,

$$
\overline{X^{(+)}}=\overline{\pi_{X}\left(X^{(+)}\right)}=\overline{\bar{X}^{(+)}}=\bar{X}^{+}
$$

as required. A dual argument shows that $\bar{X}^{*}=\overline{X^{(*)}}$. Finally, we have shown that pruning is a surjective semigroup morphism of monoids, so it must preserve the identity. Thus, it is a $(2,1,1,0)$-morphism.

From Theorem 4.5 we deduce immediately that the $(2,1,1,0)$-algebra $T^{1}(\Sigma)$ inherits a number of properties which were obvious in $U T^{1}(\Sigma)$ but perhaps less so in $T^{1}(\Sigma)$.

Corollary 4.6. Pruned multiplication is an associative operation on $T^{1}(\Sigma)$. The unary operations $*$ and + are idempotent; the subsemigroup generated by their images is commutative. 

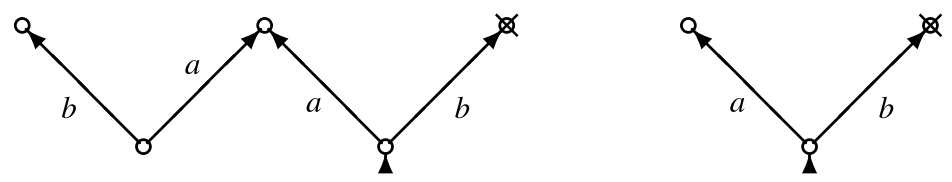

Figure 3. The trees $\left(a \times\left(b^{(+)} \times a\right)^{(*)}\right)^{(+)} \times b$ and $a^{(+)} \times b=a^{+} b$.

As well as providing a theoretical underpinning for what we wish to do, Theorem 4.5 is extremely useful for computational purposes; it means that complex expressions involving pruned trees in $T^{1}(\Sigma)$ can be computed by first evaluating them in $U T^{1}(\Sigma)$ using unpruned operations and then pruning the resulting tree only at the end. Since pruning is the hardest part of such a computation, this can result in significant efficiency savings.

Figure 3 shows some more examples of $\{a, b\}$-trees, namely the elements of $U T^{1}(\{a, b\})$ corresponding to the unpruned expressions

$$
\left(a \times\left(b^{(+)} \times a\right)^{(*)}\right)^{(+)} \times b \text { and } a^{(+)} \times b
$$

respectively. Notice that the right-hand tree is pruned, while left-hand tree admits a retract isomorphic to the right-hand tree. This means that

$$
\left(a\left(b^{+} a\right)^{*}\right)^{+} b=\overline{\left(a \times\left(b^{(+)} \times a\right)^{(*)}\right)^{(+)} \times b}=\overline{a^{(+)} \times b}=a^{+} b
$$

in the monoid $T^{1}(\{a, b\})$. We shall see later that $T^{1}(\{a, b\})$ is actually a free adequate monoid, freely generated by the base trees, so it follows that the identity $\left(A\left(B^{+} A\right)^{*}\right)^{+} B=A^{+} B$ holds in every adequate monoid and semigroup. (The reader may wish to try verifying this directly from the axioms for adequate semigroups.)

Our next objective is to establish some algebraic properties of the $(2,1,1,0)$-algebra $T^{1}(\Sigma)$ of pruned trees over a given alphabet $\Sigma$.

Proposition 4.7. Let $X \in T^{1}(\Sigma)$. Then $X^{+} X=X=X X^{*}$.

Proof. We prove that $X^{+} X=X$, the claim that $X X^{*}=X$ being dual. By Theorem 4.5,

$$
X^{+} X=\overline{X^{(+)} \times X} \text {. }
$$

Consider the unpruned product $X^{(+)} \times X$. It follows straight from the definitions of unpruned operations that this consists of two copies $\left(X_{1}\right.$ and $X_{2}$, say) of the tree $X$, with their start vertices identified, and with start vertex this start vertex and end vertex the end vertex of $X_{1}$. Define a map

$$
\pi: X^{(+)} \times X \rightarrow X^{(+)} \times X
$$

which fixes $X_{1}$ and maps each edge (vertex) of $X_{2}$ onto the corresponding edge (vertex) of $X_{1}$. Then $\pi$ is a retraction of $X^{(+)} \times X$ with image $X_{1}$ which is isomorphic to $X$. Hence, by Proposition 3.5,

$$
\overline{X^{(+)} \times X}=\overline{\pi\left(X^{(+)} \times X\right)}=\bar{X} .
$$


The following proposition justifies the name we have given to idempotent trees.

Proposition 4.8. For any $X \in T^{1}(\Sigma)$ the following are equivalent:

(i) $X$ is an idempotent tree;

(ii) $X$ is an idempotent element under pruned multiplication;

(iii) $X=X^{+}$;

(iv) $X=Y^{+}$for some $Y \in T^{1}(\Sigma)$;

(v) $X=X^{*}$;

(vi) $X=Y^{*}$ for some $Y \in T^{1}(\Sigma)$.

Proof. We prove the equivalence of (i), (ii), (iii) and (iv), the equivalence of (i), (ii), (v) and (vi) being dual. That (iii) implies (iv) is immediate. That (iv) implies (i) and that (i) implies (iii) follow directly from the definition of the + operation. If (iii) holds, so that $X=X^{+}$, then, by Proposition 4.7,

$$
X X=X^{+} X=X
$$

so that (ii) holds.

To complete the proof we shall show that (ii) implies (i). Indeed, suppose for a contradiction that (ii) holds, that is, that $X$ is idempotent under pruned multiplication, but that (i) does not, so that $X$ has distinct start and end vertices, and hence at least one trunk edge. Let $n$ be the number of trunk edges in $X$. Then $X \times X$ has $2 n$ trunk edges and, since pruning fixes the trunk, so does $X X=\overline{X \times X}$. But since $n>0$ we have $2 n \neq n$, so this contradicts the fact that $X$ is idempotent under pruned multiplication. This completes the proof that (ii) implies (i).

The following proposition shows that each pruned tree $X \in T(\Sigma)$ is $\mathcal{L}^{*}$-related (respectively, $\mathcal{R}^{*}$-related) to the idempotent $X^{*}$ (respectively, $X^{+}$), and hence that $T^{1}(\Sigma)$ is abundant.

Proposition 4.9. Let $A, B, X \in T^{1}(\Sigma)$. Then:

- $A X=B X$ if and only if $A X^{+}=B X^{+}$; and

- $X A=X B$ if and only if $X^{*} A=X^{*} B$.

Proof. We show that $A X=B X$ if and only if $A X^{+}=B X^{+}$, the other claim being dual. Certainly if $A, B$ and $X$ are pruned trees such that $A X^{+}=B X^{+}$then, by Proposition 4.7,

$$
A X=A\left(X^{+} X\right)=\left(A X^{+}\right) X=\left(B X^{+}\right) X=B\left(X^{+} X\right)=B X .
$$

Conversely, suppose that $A X=B X$; we must show that $A X^{+}=B X^{+}$. Let $\pi_{A}: A \times X \rightarrow$ $A \times X$ and $\pi_{B}: B \times X \rightarrow B \times X$ be retractions with images isomorphic to

$$
\overline{A \times X}=A X=B X=\overline{B \times X} \text {. }
$$

Now by the definition of unpruned operations, the tree $A \times X^{(+)}$has the same underlying graph as $A \times X$ and the same start vertex, but with end vertex at the start vertex of $X$ instead of the end vertex of $X$. Since the latter vertex lies in the trunk 
of $A \times X$ it is fixed by $\pi_{A}$, and it follows that $\pi_{A}$ also defines a retraction of $A \times X^{(+)}$; its image has the same underlying graph as $A X=B X$ but with end vertex moved to the start vertex of $X$. Similarly, $\pi_{B}$ defines a retraction of $B \times X^{(+)}$; its image also has the same underlying graph as $A X=B X$ but with end vertex at the start vertex of $X$. Now, using Proposition 3.5 and Theorem 4.5,

$$
A X^{+}=\overline{A \times X^{(+)}}=\overline{\pi_{A}\left(A \times X^{(+)}\right)}=\overline{\pi_{B}\left(B \times X^{(+)}\right)}=\overline{B \times X^{(+)}}=B X^{+} .
$$

We are now ready to prove our second main theorem.

Theorem 4.10. Let $\Sigma$ be an alphabet. Then $T^{1}(\Sigma)$ is an adequate monoid under the operations of pruned multiplication, + and $*$.

Proof. For any element $X \in T^{1}(\Sigma)$, by Proposition 4.9 we have $X \mathcal{R}^{*} X^{+}$, where by Proposition 4.8 the element $X^{+}$is idempotent under pruned multiplication; hence $T^{1}(\Sigma)$ is left abundant. Moreover, by Proposition 4.8 again, the idempotents under pruned multiplication are exactly the elements of the form $X^{+}$, which by Corollary 4.6 commute. Thus, $T^{1}(\Sigma)$ is left adequate. A dual argument shows that $T^{1}(\Sigma)$ is right adequate, so it follows that it is adequate.

\section{The free adequate monoid and semigroup}

We saw in the previous section that, for any alphabet $\Sigma$, the $(2,1,1,0)$-algebra $T^{1}(\Sigma)$ is an adequate monoid. In this section we shall show that it is in fact a free adequate monoid, freely generated by the subset $\Sigma$ of base trees. By Proposition 2.2 this also establishes that $T(\Sigma)$ is the free adequate semigroup on $\Sigma$.

To keep the proofs in this section concise, we shall need some additional notation. If $X$ is a tree and $S$ is a set of nontrunk edges and vertices of $X$ then $X \backslash S$ denotes the largest subtree of $X$ (recalling that a subtree must be connected and contain the start and end vertices, and hence the trunk) which does not contain any vertices or edges from $S$. If $s$ is a single edge or vertex we write $X \backslash s$ for $X \backslash\{s\}$. If $u$ and $v$ are vertices of $X$ such that there is a directed path from $u$ to $v$ then we shall denote by $\left.X\right|_{v} ^{u}$ the tree which has the same underlying labelled directed graph as $X$ but start vertex $u$ and end vertex $v$. If $X$ has start vertex $a$ and end vertex $b$ then we define $\left.X\right|^{u}=\left.X\right|_{b} ^{u}$ and $\left.X\right|_{v}=\left.X\right|_{v} ^{a}$ where applicable. Thus, for example, $\left.X\right|_{v} ^{v} \backslash e$ means the largest connected subgraph of $X$ containing the vertex $v$ but not the edge $e$, viewed as an idempotent tree with start and end vertex $v$.

Proposition 5.1. The semigroup $U T^{1}(\Sigma)$ of unpruned trees is generated as a $(2,1,1,0)$-algebra by the set $\Sigma$ of base trees.

Proof. Let $\langle\Sigma\rangle$ denote the $(2,1,1,0)$-subalgebra of $U T^{1}(\Sigma)$ generated by $\Sigma$. We wish to show that every tree in $U T^{1}(\Sigma)$ is contained in $\langle\Sigma\rangle$. We proceed by induction on number of edges. The tree with no edges is the identity element of $U T^{1}(\Sigma)$ and so by definition is contained in every $(2,1,1,0)$-subalgebra of $U T^{1}(\Sigma)$, and in particular 
in $\langle\Sigma\rangle$. Now suppose for induction that $X \in U T^{1}(\Sigma)$ has at least one edge, and that every tree in $U T^{1}(\Sigma)$ with strictly fewer edges lies in $\langle\Sigma\rangle$.

First suppose that $X$ has at least one trunk edge. Let $v_{0}$ be the start vertex of $X$, let $e$ be the trunk edge incident with $v_{0}$, let $a$ be the label of $e$ and let $v_{1}$ be the vertex at the end of $e$, that is, the second trunk vertex of $X$. Let $Y=\left.X\right|_{v_{0}} ^{v_{0}} \backslash e$ and $Z=\left.X\right|^{v_{1}} \backslash e$. Then $Y$ and $Z$ are pruned trees with strictly fewer edges than $X$, and so by induction lie in $\langle\Sigma\rangle$, and clearly, from the definitions, $Y \times a \times Z=X$ so that $X \in\langle\Sigma\rangle$ as required.

Next suppose that $X$ has no trunk edges. Let $e$ be any edge incident with the start vertex $v_{0}$, and suppose that $e$ has label $a$. Let $v_{1}$ be the vertex at the other end of $e$ from $v_{0}$. Define $Y=\left.X\right|_{v_{0}} ^{v_{0}} \backslash e$ and $Z=\left.X\right|_{v_{1}} ^{v_{1}} \backslash e$. Then $Y$ and $Z$ are pruned trees with strictly fewer edges than $X$, and so by the inductive hypothesis lie in $\langle\Sigma\rangle$. If $e$ is orientated away from the start vertex of $X$, then, from the definitions of unpruned operations, $X=Y \times(a \times Z)^{(+)}$. Similarly, if $e$ is orientated towards the start vertex then $(Z \times a)^{(*)} \times Y=X$, thus establishing in all cases that $X \in\langle\Sigma\rangle$.

Combining this with Theorem 4.5 gives the following immediate corollary.

Corollary 5.2. The semigroup $T^{1}(\Sigma)$ of pruned trees is generated as a $(2,1,1,0)$ algebra by the set $\Sigma$ of base trees.

Now suppose that $M$ is an adequate monoid and $\chi: \Sigma \rightarrow M$ is a function. Recall that $\Sigma$ is identified with the set of (isomorphism types of) base trees in $U T^{1}(\Sigma)$ and $T^{1}(\Sigma)$. Our objective is to show that there is a unique $(2,1,1,0)$-morphism from $T^{1}(\Sigma)$ to $M$ which extends the function $\chi$.

Let $E(M)$ denote the semilattice of idempotents of the adequate monoid $M$. We begin by defining a map $\tau: U E^{1}(\Sigma) \rightarrow E(M)$ from the set $U E^{1}(\Sigma)$ of (not necessarily pruned) idempotent trees to $E(M)$. Let $X$ be an idempotent tree. If $X$ has no edges then we define $\tau(X)=1$. Otherwise, we define $\tau(X)$ recursively, in terms of the value of $\tau$ on idempotent trees with strictly fewer edges than $X$, as follows.

Let $v$ be the start vertex of $X$ (which, since $X$ is an idempotent tree, is also the end vertex of $X)$. Let $E^{+}(X)$ be the set of edges in $X$ which start at $v$, and $E^{-}(X)$ the set of edges in $X$ which end at $v$. Now we define

$$
\tau(X)=\left(\prod_{e \in E^{+}(X)}\left[\chi(\lambda(e)) \tau\left(\left.X\right|_{\omega(e)} ^{\omega(e)} \backslash e\right)\right]^{+}\right)\left(\prod_{e \in E^{-}(X)}\left[\tau\left(\left.X\right|_{\alpha(e)} ^{\alpha(e)} \backslash e\right) \chi(\lambda(e))\right]^{*}\right) .
$$

Notice that since $X$ has at least one edge and is connected, this product cannot be empty. Notice also that, since all the factors are idempotent and idempotents commute in the adequate semigroup $M$, the value of this product is idempotent, and is independent of the order in which the factors are multiplied. The value clearly depends only on the isomorphism type of $X$, and so the map $\tau$ is a well-defined function from $U E^{1}(\Sigma)$ to $E(M)$. We now establish some of its elementary properties.

Proposition 5.3. Let $X$ be an idempotent tree with start (and end) vertex $v$, and let $X_{1}$ and $X_{2}$ be subtrees of $X$ such that $X=X_{1} \cup X_{2}$ and $X_{1} \cap X_{2}=\{v\}$. Then $\tau(X)=\tau\left(X_{1}\right) \tau\left(X_{2}\right)$. 
Proof. Clearly $E^{+}(X)=E^{+}\left(X_{1}\right) \cup E^{+}\left(X_{2}\right)$ and, for $i \in\{1,2\}$ and $e \in E^{+}\left(X_{i}\right)$,

$$
\tau\left(\left.X\right|_{\omega(e)} ^{\omega(e)} \backslash e\right)=\tau\left(\left.X_{i}\right|_{\omega(e)} ^{\omega(e)} \backslash e\right),
$$

so it follows that

$$
\left[\chi(\lambda(e)) \tau\left(\left.X\right|_{\omega(e)} ^{\omega(e)} \backslash e\right)\right]^{+}=\left[\chi(\lambda(e)) \tau\left(\left.X_{i}\right|_{\omega(e)} ^{\omega(e)} \backslash e\right)\right]^{+} .
$$

A dual claim holds for $e \in E^{-}\left(X_{i}\right)$ and the claim then follows directly from the definition of $\tau$.

Corollary 5.4. Let $X$ be an idempotent tree with start vertex $v$. If $e$ is an edge with $\alpha(e)=v$ then

$$
\tau(X)=\tau(X \backslash \omega(e))\left[\chi(\lambda(e)) \tau\left(\left.X\right|_{\omega(e)} ^{\omega(e)} \backslash e\right)\right]^{+},
$$

while if $e$ is an edge with $\omega(e)=v$ then

$$
\tau(X)=\tau(X \backslash \alpha(e))\left[\tau\left(\left.X\right|_{\alpha(e)} ^{\alpha(e)} \backslash e\right) \chi(\lambda(e))\right]^{*} .
$$

Proof. We prove the claim in the case where $\alpha(e)=v$, the case where $\omega(e)=v$ being dual. Let $X_{1}=X \backslash e=X \backslash \omega(e)$, let $S$ be the set of edges in $X$ which are incident with $v$ and let $X_{2}=X \backslash(S \backslash\{e\})$ be the maximum subtree of $X$ containing $e$ but none of the other edges incident with $v$. Now clearly $E^{+}\left(X_{2}\right)=\{e\}$ and $E^{-}\left(X_{2}\right)=\emptyset$ so, by the definition of $\tau$,

$$
\tau\left(X_{2}\right)=\left[\chi(\lambda(e)) \tau\left(\left.X\right|_{\omega(e)} ^{\omega(e)} \backslash e\right)\right]^{+} .
$$

Also $X=X_{1} \cup X_{2}$ and $X_{1} \cap X_{2}=\{v\}$ so, by Proposition 5.3,

$$
\tau(X)=\tau\left(X_{1}\right) \tau\left(X_{2}\right)=\tau(X \backslash \omega(e))\left[\chi(\lambda(e)) \tau\left(\left.X\right|_{\omega(e)} ^{\omega(e)} \backslash e\right)\right]^{+}
$$

as required.

Next we define a map $\rho: U T^{1}(\Sigma) \rightarrow M$, from the set of isomorphism types of (not necessarily pruned) $\Sigma$-trees to the adequate monoid $M$. Suppose that a tree $X$ has trunk vertices $v_{0}, \ldots, v_{n}$ in sequence. For $1 \leq i \leq n$, let $a_{i}$ be the label of the edge from $v_{i-1}$ to $v_{i}$. For $0 \leq i \leq n$, let

$$
X_{i}=\left.X\right|_{v_{i}} ^{v_{i}} \backslash \theta(X),
$$

that is, $X_{i}$ is the maximum connected subgraph of $X$ containing $v_{i}$ but no trunk edges, viewed as an idempotent tree with start and end vertex $v_{i}$. Then we define

$$
\rho(X)=\tau\left(X_{0}\right) \chi\left(a_{1}\right) \tau\left(X_{1}\right) \chi\left(a_{2}\right) \cdots \chi\left(a_{n-1}\right) \tau\left(X_{n-1}\right) \chi\left(a_{n}\right) \tau\left(X_{n}\right) .
$$

The value of $\rho$ clearly depends only on the isomorphism type of $X$ so $\rho$ is indeed a welldefined map on $U T^{1}(\Sigma)$. Notice also that if $X$ is an idempotent tree then $\rho(X)=\tau(X)$. We now establish some elementary properties of the map $\rho$. 
Proposition 5.5. Let $X$ be a tree with trunk vertices $v_{0}, \ldots, v_{n}$ in sequence, where $n \geq 1$. Let $a_{1}$ and $a_{n}$ be the labels of the edges from $v_{0}$ to $v_{1}$ and from $v_{n-1}$ to $v_{n} m$ respectively. Then

$$
\rho(X)=\tau\left(\left.X\right|_{v_{0}} ^{v_{0}} \backslash v_{1}\right) \chi\left(a_{1}\right) \rho\left(\left.X\right|^{v_{1}} \backslash v_{0}\right)=\rho\left(\left.X\right|_{v_{n-1}} \backslash v_{n}\right) \chi\left(a_{n}\right) \tau\left(\left.X\right|_{v_{n}} ^{v_{n}} \backslash v_{n-1}\right) .
$$

Proof. We prove the first equality, the remaining part being dual. Let $X_{0}, \ldots, X_{n}$ be as in the definition of $\rho$, so that

$$
\rho(X)=\tau\left(X_{0}\right) \chi\left(a_{1}\right) \tau\left(X_{1}\right) \chi\left(a_{2}\right) \cdots \chi\left(a_{n-1}\right) \tau\left(X_{n-1}\right) \chi\left(a_{n}\right) \tau\left(X_{n}\right) .
$$

It follows straight from the definition that

$$
\rho\left(\left.X\right|^{v_{1}} \backslash v_{0}\right)=\tau\left(X_{1}\right) \chi\left(a_{2}\right) \cdots \chi\left(a_{n-1}\right) \tau\left(X_{n-1}\right) \chi\left(a_{n}\right) \tau\left(X_{n}\right),
$$

so

$$
\begin{aligned}
\rho(X) & =\tau\left(X_{0}\right) \chi\left(a_{1}\right) \rho\left(\left.X\right|^{v_{1}} \backslash v_{0}\right) \\
& =\tau\left(\left.X\right|_{v_{0}} ^{v_{0}} \backslash v_{1}\right) \chi\left(a_{1}\right) \rho\left(\left.X\right|^{v_{1}} \backslash v_{0}\right)
\end{aligned}
$$

as required.

The next proposition says that the map $\rho$ is actually a well-defined $(2,1,1,0)$ morphism from the monoid of isomorphism types of $\Sigma$-trees (with unpruned operations) to the adequate monoid $M$. Later, we shall see that it even induces a welldefined map from the monoid of isomorphism types of pruned $\Sigma$-trees (with pruned operations) to $M$.

Proposition 5.6. The map $\rho: U T^{1}(\Sigma) \rightarrow M$ is a morphism of $(2,1,1,0)$-algebras.

Proof. Let $X$ and $Y$ be trees, say with trunk vertices $u_{0}, \ldots, u_{m}$ and $v_{0}, \ldots, v_{n}$ in sequence, respectively. For each $1 \leq i \leq m$ let $a_{i}$ be the label of the edge from $u_{i-1}$ to $u_{i}$, and for each $1 \leq i \leq n$ let $b_{i}$ be the label of the edge from $v_{i-1}$ to $v_{i}$. For each $0 \leq i \leq m$ let $X_{i}=\left.X\right|_{u_{i}} ^{u_{i}} \backslash \theta(X)$, and similarly for each $0 \leq i \leq n$ define $Y_{i}=\left.Y\right|_{v_{i}} ^{v_{i}} \backslash \theta(Y)$.

Consider now the unpruned product $X \times Y$. It is easily seen that for $0 \leq i<m$,

$$
\left.(X \times Y)\right|_{u_{i}} ^{u_{i}} \backslash \theta(X \times Y)=X_{i},
$$

while for $0<i \leq n$,

$$
\left.(X \times Y)\right|_{v_{i}} ^{v_{i}} \backslash \theta(X \times Y)=Y_{i} .
$$

Considering now the remaining trunk vertex $u_{m}=v_{0}$ of $X \times Y$,

$$
\left.(X \times Y)\right|_{u_{m}} ^{u_{m}} \backslash \theta(X \times Y)=\left.(X \times Y)\right|_{v_{0}} ^{v_{0}} \backslash \theta(X \times Y)=X_{m} \times Y_{0}
$$

By Proposition 5.3 and the definition of unpruned multiplication, $\tau\left(X_{m} \times Y_{0}\right)=$ $\tau\left(X_{m}\right) \tau\left(Y_{0}\right)$. So, using the definition of $\rho$,

$$
\begin{aligned}
\rho(X \times Y) & =\tau\left(X_{0}\right) \chi\left(a_{1}\right) \tau\left(X_{1}\right) \cdots \chi\left(a_{m}\right) \tau\left(X_{m} \times Y_{0}\right) \chi\left(b_{1}\right) \tau\left(Y_{1}\right) \chi\left(b_{2}\right) \cdots \chi\left(b_{n}\right) \tau\left(Y_{n}\right) \\
& =\tau\left(X_{0}\right) \chi\left(a_{1}\right) \tau\left(X_{1}\right) \cdots \chi\left(a_{m}\right) \tau\left(X_{m}\right) \tau\left(Y_{0}\right) \chi\left(b_{1}\right) \tau\left(Y_{1}\right) \chi\left(b_{2}\right) \cdots \chi\left(b_{n}\right) \tau\left(Y_{n}\right) \\
& =\rho(X) \rho(Y) .
\end{aligned}
$$


Next we claim that $\rho\left(X^{(+)}\right)=\rho(X)^{+}$. We prove this by induction on the number of trunk edges in $X$. If $X$ has no trunk edges then $X=X^{(+)}$and so, using the fact that $\tau(X) \in E(M)$ is fixed by the + operation in $M$,

$$
\rho\left(X^{(+)}\right)=\rho(X)=\tau(X)=\tau(X)^{+}=\rho(X)^{+} .
$$

Now suppose for induction that $X$ has at least one trunk edge and that the claim holds for trees with strictly fewer trunk edges. Recall that

$$
X_{0}=\left.X\right|_{u_{0}} ^{u_{0}} \backslash \theta(X)=\left.X\right|_{u_{0}} ^{u_{0}} \backslash u_{1}
$$

and let $Z=\left.X\right|^{u_{1}} \backslash u_{0}$. Now

$$
\begin{aligned}
\rho\left(X^{(+)}\right) & \left.=\tau\left(X^{(+)}\right) \quad \text { (by the definition of } \rho\right) \\
& =\tau\left(X_{0}\right)\left[\chi\left(a_{1}\right) \tau\left(Z^{(+)}\right)\right]^{+} \quad \text { (by Corollary 5.4) } \\
& \left.=\tau\left(X_{0}\right)\left[\chi\left(a_{1}\right) \rho\left(Z^{(+)}\right)\right]^{+} \quad \text { (by the definition of } \rho\right) \\
& =\tau\left(X_{0}\right)\left[\chi\left(a_{1}\right) \rho(Z)^{+}\right]^{+} \quad \text { (by the inductive hypothesis) } \\
& =\tau\left(X_{0}\right)\left[\chi\left(a_{1}\right) \rho(Z)\right]^{+} \quad \text { (by Proposition 2.1(ii)) } \\
& =\left[\tau\left(X_{0}\right) \chi\left(a_{1}\right) \rho(Z)\right]^{+} \quad \text { (by Proposition 2.1(iv)) } \\
& =\rho(X)^{+} \quad \text { (by Proposition 5.5) }
\end{aligned}
$$

as required.

A dual argument shows that $\rho\left(X^{(*)}\right)=\rho(X)^{*}$. Finally, it follows directly from the definition that $\rho$ maps the identity element in $U T^{1}(\Sigma)$ (that is, the isomorphism type of the trivial tree) to the identity of $M$, and so is a $(2,1,1,0)$-morphism.

Our next objective is to establish some technical lemmas involving the function $\tau$.

Lemma 5.7. Suppose that $Y$ is an idempotent tree with start vertex $u$ and an edge from $u$ to $v$ with label $a$. Let $Y^{\prime}=\left.Y\right|_{v} ^{v}$. Then $\tau(Y)\left[\chi(a) \tau\left(Y^{\prime}\right)\right]^{+}=\tau(Y)$ and $[\tau(Y) \chi(a)]^{*} \tau\left(Y^{\prime}\right)=$ $\tau\left(Y^{\prime}\right)$ in $M$.

Proof. We prove the second claim, the first being dual. Let $Y_{1}=Y \backslash v$ and $Y_{2}=Y^{\prime} \backslash u$. For readability, we let $B=\tau\left(Y_{1}\right), C=\tau\left(Y_{2}\right)$ and $x=\chi(a)$, noting that $B$ and $C$ are idempotent. Now from Corollary 5.4 and the definition of $\tau$,

$$
\tau(Y)=\tau\left(Y_{1}\right)\left(\chi(a) \tau\left(Y_{2}\right)\right)^{+}=B(x C)^{+},
$$

while

$$
\tau\left(Y^{\prime}\right)=\left(\tau\left(Y_{1}\right) \chi(a)\right)^{*} \tau\left(Y_{2}\right)=(B x)^{*} C
$$


So now

$$
\begin{aligned}
{[\tau(Y) \chi(a)]^{*} \tau\left(Y^{\prime}\right) } & =\left[B(x C)^{+} x\right]^{*}(B x)^{*} C \\
& =\left[B(x C)^{+} x(B x)^{*} C\right]^{*} \quad \text { (by Proposition 2.1(iv)) } \\
& =\left[(x C)^{+} B x(B x)^{*} C\right]^{*} \quad \text { (since idempotents commute) } \\
& =\left[(x C)^{+} B x C\right]^{*} \quad \text { (by Proposition 2.1(iii)) } \\
& =\left[B(x C)^{+} x C\right]^{*} \quad \text { (since idempotents commute) } \\
& =[B x C]^{*} \quad(\text { by Proposition 2.1(iii) }) \\
& =(B x)^{*} C \quad(\text { by Proposition 2.1(iv) }) \\
& =\tau\left(Y^{\prime}\right)
\end{aligned}
$$

as required.

Lemma 5.8. Let $Y$ be an idempotent tree with start vertex $u$ and suppose that $Y$ has an edge from $u$ to $v$ with label $a$. Let $Y^{\prime}=\left.Y\right|_{v} ^{v}$ and suppose that $e \in E(M)$ is an idempotent such that $\tau\left(Y^{\prime}\right) e=\tau\left(Y^{\prime}\right)$. Then $\tau(Y)(\chi(a) e)^{+}=\tau(Y)$.

Proof. First notice that, by Proposition 2.1(vi),

$$
\left[\chi(a) \tau\left(Y^{\prime}\right)\right]^{+}(\chi(a) e)^{+}=\left[\chi(a) \tau\left(Y^{\prime}\right)\right]^{+} .
$$

Now

$$
\begin{aligned}
\tau(Y)(\chi(a) e)^{+} & =\tau(Y)\left[\chi(a) \tau\left(Y^{\prime}\right)\right]^{+}(\chi(a) e)^{+} \quad \text { (by Lemma 5.7) } \\
& =\tau(Y)\left[\chi(a) \tau\left(Y^{\prime}\right)\right]^{+} \quad \text { (by the observation above) } \\
& =\tau(Y) \quad \text { (by Lemma } 5.7 \text { again). }
\end{aligned}
$$

Lemma 5.9. Suppose that $\mu: X \rightarrow Y$ is a morphism of idempotent $\Sigma$-trees. Then $\tau(Y) \tau(X)=\tau(Y)$.

Proof. We use induction on the number of edges in $X$. If $X$ has no edges then $\tau(X)=1$ and the result is clear. Now suppose that $X$ has at least one edge and for induction that the result holds for trees $X$ with fewer edges. By the definition of $\tau$,

$$
\tau(X)=\left(\prod_{e \in E^{+}(X)}\left[\chi(\lambda(e)) \tau\left(\left.X\right|_{\omega(e)} ^{\omega(e)} \backslash e\right)\right]^{+}\right)\left(\prod_{e \in E^{-}(X)}\left[\tau\left(\left.X\right|_{\alpha(e)} ^{\alpha(e)} \backslash e\right) \chi(\lambda(e))\right]^{*}\right)
$$

while

$$
\tau(Y)=\left(\prod_{e \in E^{+}(Y)}\left[\chi(\lambda(e)) \tau\left(\left.Y\right|_{\omega(e)} ^{\omega(e)} \backslash e\right)\right]^{+}\right)\left(\prod_{e \in E^{-}(Y)}\left[\tau\left(\left.Y\right|_{\alpha(e)} ^{\alpha(e)} \backslash e\right) \chi(\lambda(e))\right]^{*}\right) .
$$

Suppose now that $e \in E^{+}(X)$. Then since $\mu$ preserves endpoints and maps the start vertex of $X$ to the start vertex of $Y$, the edge $\mu(e)$ lies in $E^{+}(Y)$. We claim that the 
factor corresponding to $e$ in the above expression for $\tau(X)$ is absorbed into $\tau(Y)$. First notice that $\left.X\right|_{\omega(e)} ^{\omega(e)} \backslash e$ has strictly fewer edges than $X$, and the restriction of $\mu$ to this subtree is a morphism to $Y^{\prime}=\left.Y\right|_{\mu(\omega(e))} ^{\mu(\omega(e))}$. Hence, by the inductive hypothesis, $\tau\left(Y^{\prime}\right) \tau\left(\left.X\right|_{\omega(e)} ^{\omega(e)} \backslash e\right)=\tau\left(Y^{\prime}\right)$. Now since $\mu(e)$ is an edge from $\mu(v)$ to $\mu(\omega(e))$ with label $\lambda(e)$, by Lemma 5.8 , it follows that

$$
\tau(Y)\left[\chi(\lambda(e)) \tau\left(\left.X\right|_{\omega(e)} ^{\omega(e)} \backslash e\right)\right]^{+}=\tau(Y)
$$

as required.

A dual argument shows that factors in the product expression for $\tau(X)$ resulting from edges in $E^{-}(X)$ are also absorbed into $\tau(Y)$. Thus, $\tau(Y) \tau(X)=\tau(Y)$ as required.

Corollary 5.10. Let $Y$ be an idempotent tree and $X$ be a subtree of $Y$. Then $\tau(Y) \tau(X)=\tau(Y)$.

Proof. The embedding of $X$ into $Y$ satisfies the conditions of Lemma 5.9.

Corollary 5.11. Let $Y$ be a retract of an idempotent tree $X$. Then $\tau(X)=\tau(Y)$.

Proof. Let $\pi: X \rightarrow X$ be a retraction of $X$ onto $Y$. Since $\pi$ is a morphism, Lemma 5.9 tells us that $\tau(X) \tau(\pi(X))=\tau(\pi(X))=\tau(Y)$. But since $Y=\pi(X)$ is a subtree of $X$, Corollary 5.10 yields $\tau(X) \tau(\pi(X))=\tau(X)$.

We now turn our attention to the function $\rho$.

Lemma 5.12. Let $X$ be a tree with trunk vertices $v_{0}, \ldots, v_{n}$ in sequence. Then $\rho(X)=$ $\rho(X) \tau\left(\left.X\right|_{v_{n}} ^{v_{n}}\right)$.

Proof. Let $X^{\prime}=\left.X\right|_{v_{n}} ^{v_{n}}$. We use induction on the number of trunk edges in $X$. Clearly, if $X$ has no trunk edges then $X=X^{\prime}$ and from the definition of $\rho$ we have $\rho(X)=\tau\left(X^{\prime}\right)$, so the claim reduces to the fact that $\tau\left(X^{\prime}\right)$ is idempotent. Now suppose that $X$ has at least one trunk edge and that the claim holds for $X$ with strictly fewer trunk edges. Let $Y=\left.X\right|_{v_{n-1}} ^{v_{0}} \backslash v_{n}$, let $Y^{\prime}=\left.Y\right|_{v_{n-1}} ^{v_{n-1}}$ and let $X_{n}=\left.X\right|_{v_{n}} ^{v_{n}} \backslash v_{n-1}$. Let $a_{n}$ be the label of the edge from $v_{n-1}$ to $v_{n}$. By Corollary 5.4,

$$
\tau\left(X^{\prime}\right)=\tau\left(X_{n}\right)\left[\tau\left(Y^{\prime}\right) \chi\left(a_{n}\right)\right]^{*} .
$$

Now by Proposition 5.5 we deduce that $\rho(X)=\rho(Y) \chi\left(a_{n}\right) \tau\left(X_{n}\right)$. Also, by the inductive hypothesis, $\rho(Y)=\rho(Y) \tau\left(Y^{\prime}\right)$. Putting these observations together,

$$
\begin{aligned}
\rho(X) \tau\left(X^{\prime}\right) & =\left(\rho(Y) \chi\left(a_{n}\right) \tau\left(X_{n}\right)\right)\left(\tau\left(X_{n}\right)\left[\tau\left(Y^{\prime}\right) \chi\left(a_{n}\right)\right]^{*}\right) \\
& =\left[\rho(Y) \tau\left(Y^{\prime}\right)\right] \chi\left(a_{n}\right) \tau\left(X_{n}\right)\left[\tau\left(Y^{\prime}\right) \chi\left(a_{n}\right)\right]^{*} \\
& =\rho(Y)\left[\tau\left(Y^{\prime}\right) \chi\left(a_{n}\right)\right]\left[\tau\left(Y^{\prime}\right) \chi\left(a_{n}\right)\right]^{*} \tau\left(X_{n}\right) \\
& =\rho(Y) \tau\left(Y^{\prime}\right) \chi\left(a_{n}\right) \tau\left(X_{n}\right) \\
& =\rho(Y) \chi\left(a_{n}\right) \tau\left(X_{n}\right) \\
& =\rho(X)
\end{aligned}
$$

as required. 
Lemma 5.13. Let $X$ be a tree with trunk vertices $v_{0}, \ldots, v_{n}$ in sequence, and for $1 \leq i \leq n$ let $a_{i}$ be the label of the edge from $v_{i-1}$ to $v_{i}$. Then

$$
\rho(X)=\tau\left(\left.X\right|_{v_{0}} ^{v_{0}}\right) \chi\left(a_{1}\right) \tau\left(\left.X\right|_{v_{1}} ^{v_{1}}\right) \chi\left(a_{2}\right) \cdots \tau\left(\left.X\right|_{v_{n-1}} ^{v_{n-1}}\right) \chi\left(a_{n}\right) \tau\left(\left.X\right|_{v_{n}} ^{v_{n}}\right) .
$$

Proof. For $0 \leq i \leq n$, let $X_{i}=\left.X\right|_{v_{i}} ^{v_{i}} \backslash \theta(X)$ and let $X^{i}=\left.X\right|_{v_{i}} ^{v_{i}}$. Recall that, by definition,

$$
\rho(X)=\tau\left(X_{0}\right) \chi\left(a_{1}\right) \tau\left(X_{1}\right) \chi\left(a_{2}\right) \cdots \chi\left(a_{n-1}\right) \tau\left(X_{n-1}\right) \chi\left(a_{n}\right) \tau\left(X_{n}\right),
$$

so our task is to show that each term $\tau\left(X_{i}\right)$ can be replaced with $\tau\left(X^{i}\right)$.

Once again we use induction on the number of trunk edges in $X$. If $X$ has no trunk edges then $X^{0}=X=X_{0}$ so that $\rho(X)=\tau\left(X_{0}\right)=\tau\left(X^{0}\right)$ and the claim holds. Now suppose that $X$ has at least one trunk edge and that the claim holds for $X$ with strictly fewer trunk edges; we call this the outer inductive hypothesis to distinguish it from another to be introduced shortly. As in the previous proof, let $Y=\left.X\right|_{v_{n-1}} ^{v_{0}} \backslash v_{n}$. For $0 \leq i<n$, let $Y^{i}=\left.Y\right|_{v_{i}} ^{v_{i}}$. Observe that $Y$ has strictly fewer trunk edges than $X$. Now

$$
\begin{aligned}
\rho(X) & =\rho(X) \tau\left(X^{n}\right) \quad(\text { by Lemma 5.12) } \\
& =\rho(Y) \chi\left(a_{n}\right) \tau\left(X_{n}\right) \tau\left(X^{n}\right) \quad(\text { by Proposition 5.5) } \\
& =\rho(Y) \chi\left(a_{n}\right) \tau\left(X^{n}\right) \quad(\text { by Corollary 5.10) } \\
& =\tau\left(Y^{0}\right) \chi\left(a_{1}\right) \tau\left(Y^{1}\right) \chi\left(a_{2}\right) \cdots \tau\left(Y^{n-1}\right) \chi\left(a_{n}\right) \tau\left(X^{n}\right)
\end{aligned}
$$

by the outer inductive hypothesis. We now claim that

$$
\rho(X)=\tau\left(Y^{0}\right) \chi\left(a_{1}\right) \tau\left(Y^{1}\right) \chi\left(a_{2}\right) \cdots \tau\left(Y^{j-1}\right) \chi\left(a_{j}\right) \tau\left(X^{j}\right) \chi\left(a_{j+1}\right) \cdots \chi\left(a_{n}\right) \tau\left(X^{n}\right)
$$

for all $0 \leq j \leq n$. Having fixed the graph $X$, and hence the parameter $n$, we prove the claim by downward induction on the parameter $j$. We have just proved that the claim holds for for $j=n$, which establishes the base case. Suppose now for induction that $j<n$ and that the claim holds for greater values of $j$; this we call the inner inductive hypothesis. Let $Z=\left.X\right|_{v_{j+1}} ^{v_{j+1}} \backslash v_{j}$. Then

$$
\begin{aligned}
\tau\left(Y^{j}\right) \chi\left(a_{j+1}\right) \tau\left(X^{j+1}\right) & =\tau\left(Y^{j}\right) \chi\left(a_{j+1}\right) \tau(Z) \tau\left(X^{j+1}\right) \\
& =\tau\left(Y^{j}\right)\left[\chi\left(a_{j+1}\right) \tau(Z)\right]^{+}\left[\chi\left(a_{j+1}\right) \tau(Z)\right] \tau\left(X^{j+1}\right) \\
& =\tau\left(X^{j}\right) \chi\left(a_{j+1}\right) \tau(Z) \tau\left(X^{j+1}\right) \\
& =\tau\left(X^{j}\right) \chi\left(a_{j+1}\right) \tau\left(X^{j+1}\right)
\end{aligned}
$$

where the first and fourth equalities hold by Corollary 5.10, the second by Proposition 2.1(iii) and the third by Corollary 5.4. Now combining this with the inner inductive hypothesis,

$$
\begin{aligned}
\rho(X) & =\tau\left(Y^{0}\right) \cdots \tau\left(Y^{j-1}\right) \chi\left(a_{j}\right)\left[\tau\left(Y^{j}\right) \chi\left(a_{j+1}\right) \tau\left(X^{j+1}\right)\right] \chi\left(a_{j+2}\right) \cdots \chi\left(a_{n}\right) \tau\left(X^{n}\right) \\
& =\tau\left(Y^{0}\right) \cdots \tau\left(Y^{j-1}\right) \chi\left(a_{j}\right) \tau\left(X^{j}\right) \chi\left(a_{j+1}\right) \tau\left(X^{j+1}\right) \chi\left(a_{j+2}\right) \cdots \chi\left(a_{n}\right) \tau\left(X^{n}\right) .
\end{aligned}
$$

This completes the inner inductive step, so the claim now follows by induction. The case $j=0$ establishes the inductive step for the outer induction, and hence proves the lemma. 
Corollary 5.14. Let $X$ be a tree. Then $\rho(X)=\rho(\bar{X})$.

Proof. Let $\pi: X \rightarrow X$ be a retraction with image $\bar{X}$. Suppose that $X$ has trunk vertices $v_{0}, \ldots, v_{n}$. For $1 \leq i \leq n$, let $e_{i}$ be the edge from $v_{i-1}$ to $v_{i}$, and let $a_{i} \in \Sigma$ be the label of $e_{i}$. For $0 \leq i \leq n$, let $X_{i}=\left.X\right|_{v_{i}} ^{v_{i}} \backslash \theta(X)$ and let $X^{i}=\left.X\right|_{v_{i}} ^{v_{i}}$. Now since $\pi$ is a retraction of $X$, it fixes all trunk vertices of $X$, so it follows that $\pi$ is also a retraction of each $X^{i}$. Moreover, for each $i$, clearly $\pi\left(X^{i}\right)=\left.\pi(X)\right|_{v_{i}} ^{v_{i}}$. Thus

$$
\begin{aligned}
\rho(X) & =\tau\left(X^{0}\right) \chi\left(a_{1}\right) \tau\left(X^{1}\right) \chi\left(a_{2}\right) \cdots \tau\left(X^{n-1}\right) \chi\left(a_{n}\right) \tau\left(X^{n}\right) \\
& =\tau\left(\pi\left(X^{0}\right)\right) \chi\left(a_{1}\right) \tau\left(\pi\left(X^{1}\right)\right) \chi\left(a_{2}\right) \cdots \tau\left(\pi\left(X^{n-1}\right)\right) \chi\left(a_{n}\right) \tau\left(\pi\left(X^{n}\right)\right) \\
& =\tau\left(\left.\pi(X)\right|_{v_{0}} ^{v_{0}}\right) \chi\left(a_{1}\right) \tau\left(\left.\pi(X)\right|_{v_{1}} ^{v_{1}}\right) \chi\left(a_{2}\right) \cdots \tau\left(\left.\pi(X)\right|_{v_{n-1}} ^{v_{n-1}}\right) \chi\left(a_{n}\right) \tau\left(\left.\pi(X)\right|_{v_{n}} ^{v_{n}}\right) \\
& =\rho(\pi(X)) \\
& =\rho(\bar{X})
\end{aligned}
$$

where the first and fourth equalities follow from Lemma 5.13, the second from Corollary 5.11, the third from the definition of the $X^{i}$ and the fifth from the definition of $\pi$.

Now let $\hat{\rho}: T^{1}(\Sigma) \rightarrow M$ be the restriction of $\rho$ to the set of (isomorphism types of) pruned trees.

Corollary 5.15. The function $\hat{\rho}$ is a $(2,1,1,0)$-morphism from $T^{1}(\Sigma)$ (with pruned operations) to the adequate monoid $M$.

Proof. For any $X, Y \in T^{1}(\Sigma)$, combining Theorem 4.5, Corollary 5.14 and Proposition 5.6 yields

$$
\begin{gathered}
\hat{\rho}(X Y)=\rho(X Y)=\rho(\overline{X \times Y})=\rho(X \times Y)=\rho(X) \rho(Y)=\hat{\rho}(X) \hat{\rho}(Y), \\
\hat{\rho}\left(X^{+}\right)=\rho\left(\overline{X^{(+)}}\right)=\rho\left(X^{(+)}\right)=\rho(X)^{+}=\hat{\rho}(X)^{+}
\end{gathered}
$$

and

$$
\hat{\rho}\left(X^{*}\right)=\rho\left(\overline{X^{(*)}}\right)=\rho\left(X^{(*)}\right)=\rho(X)^{*}=\hat{\rho}(X)^{*} .
$$

Finally, that $\hat{\rho}$ maps the identity of $T^{1}(\Sigma)$ to the identity of $M$ follows directly from the definition.

We are now ready to prove our main result, which gives a concrete characterisation of the free adequate monoid on a given generating set.

Theorem 5.16. Let $\Sigma$ be a set. Then $T^{1}(\Sigma)$ is a free object in the quasivariety of adequate monoids, freely generated by the set $\Sigma$ of base trees.

Proof. By Theorem 4.10, $T^{1}(\Sigma)$ is an adequate monoid. Now for any adequate monoid $M$ and function $\chi: \Sigma \rightarrow M$, define $\hat{\rho}: T^{1}(\Sigma) \rightarrow M$ as above. By Corollary 5.15, $\hat{\rho}$ is a $(2,1,1,0)$-morphism, and it is immediate from the definitions that $\hat{\rho}(a)=\chi(a)$ for every $a \in \Sigma$, so that $\hat{\rho}$ extends $\chi$. Finally, by Corollary $5.2, \Sigma$ is a $(2,1,1,0)$-algebra generating set for $T^{1}(\Sigma)$; it follows that the morphism $\hat{\rho}$ is uniquely determined by 
its restriction to the set $\Sigma$ of base trees, and hence is the unique morphism with the claimed properties.

Combining Theorem 5.16 with Proposition 2.2, we also obtain immediately a description of the free adequate semigroup.

TheOREM 5.17. Let $\Sigma$ be a set. Then the $T(\Sigma)$ is a free object in the quasivariety of adequate semigroups, freely generated by the set $\Sigma$ of base trees.

\section{Remarks}

In this section we collect together some observations on our methods, their potential for wider application, and their connections to other work.

The 'pruning' process (that is, computing a pruned tree $\bar{X}$ from the unpruned tree $X$ ) can be more concretely realised as a process of pruning 'branches'. Look for a 'branch' of $X$ (a subgraph which contains all the vertices and edges on the nontrunkward side of some given edge) which can be 'folded' (mapped by a retraction which fixes everything except the given branch) into the rest of the tree, and remove it. Repeat this process until no such branches can be found, and one is left with $\bar{X}$.

If one drops the requirement that a $\Sigma$-tree should have a directed path from the start vertex to the end vertex and defines the pruning of a tree to be the (isomorphism type of the) minimal image under an arbitrary morphism instead of a retraction, one ends up with the Munn representation of the free inverse monoid. (Edge-labelled directed trees which do not admit nonidentity morphisms are, up to isomorphism, exactly the connected subgraphs of the Cayley graph of the free group on the labelling alphabet.)

As a consequence, the natural morphism of the free adequate semigroup onto the free ample semigroup and into the free inverse semigroup (taking $x^{+}$to $x x^{-1}$ and $x^{*}$ to $x^{-1} x$ ) has a natural description as a map of our trees onto Munn trees. Namely, one just takes the homomorphically minimal image of the tree, and embeds it into the Cayley graph of the free group with the start vertex at the identity. More concretely, this can be realised by the following process. Whenever two distinct edges have the same label and endpoint, identify the edges and their respective start vertices; dually, whenever two edges have the same label and startpoint, identify the edges and their respective endpoints. Repeat until no such identifications are possible (which must happen, since each identification reduces the number of edges). This is essentially one part (since there is no deletion of spurs) of the folding process originally developed by Stallings [23] and extensively employed and extended to solve numerous problems in combinatorial group theory.

Of course there is also a natural retraction of the free adequate monoid onto the free monoid on the same generating set, which simply maps each tree to the label of its trunk.

For the reader who prefers to think of free objects as 'word algebras' (sets of equivalence classes of formal expressions involving the generators and operations), the map $\hat{\rho}$ defined in Section 5 can of course be used to give an explicit isomorphism 
from $T^{1}(\Sigma)$ to the appropriate word algebra. This yields (up to some unimportant technicalities involving the order in which idempotents are multiplied) a normal form for elements as formal expressions. Note that the size of the expression obtained from a tree is linear in the number of vertices and edges in the tree.

Branco et al. [1, 11] have recently initiated the study of free objects in the quasivariety of left adequate monoids, as part of their theory of proper left and right adequate semigroups. It transpires that the $(2,1)$-subalgebra of $T^{1}(\Sigma)$ generated by the base trees under multiplication and + (respectively, multiplication and $*$ ) is exactly the free left adequate (right adequate) monoid on $\Sigma$. The proof, which is similar in spirit and outline to Section 5 but rather different in some of the technical details, is given in [16].

The construction in Section 5 of a morphism from $T^{1}(\Sigma)$ to an adequate monoid $M$ depends only on the facts that $M$ is associative with commuting idempotents, and that the + and $*$ operations are idempotent with idempotent images and satisfy the six properties given in the case of adequate semigroups by Proposition 2.1. Thus, the free adequate semigroups will also be free objects in any category of $(2,1,1)$-algebras which contains them and satisfy these conditions. This includes, in particular, the class of Ehresmann semigroups [17]. Similar remarks apply to free left adequate semigroups and free right adequate semigroups (which, in particular, are free objects also in the classes left Ehresmann semigroups and right Ehresmann semigroups, respectively) and to the corresponding classes of monoids.

The classes of monoids that we have studied can all be generalised in an obvious way to give corresponding classes of small categories. For example, there is a natural notion of an adequate category, the single object instances of which are exactly the adequate monoids. A natural extension of our methods can be used to describe the free adequate, free left adequate and free right adequate category generated by a given directed graph; in this case one works with directed trees in which the vertices are labelled by vertices of the generating graph, and the edges labelled by edges of the generating graph with a requirement that the vertex labels match up with the edge labels in the obvious way. Just as in the previous remark, the free left adequate category will also be the free left Ehresmann category. Left Ehresmann categories are generalisations of the restriction categories studied by Cockett and Lack [3], which in the terminology of semigroup theory are weakly left E-ample categories [12]. The generalisation of our results to categories thus relates to our main results in the same way that the description of the free restriction category on a graph given in [2] relates to the descriptions of free ample and left ample monoids given by Fountain, Gomes and Gould $[9,10]$.

\section{Applications}

In this section we show how our characterisation of the free adequate monoids and semigroup can be applied to establish some of their basic computational and algebraic properties. 
Our main theorems immediately yield a decision algorithm for the word problem in a finitely generated free adequate monoid. Given two expressions, it is a polynomial time procedure to compute the corresponding unpruned trees. The expressions are equivalent exactly if the trees admit isomorphic retracts; this can be checked by exhaustive search or, nondeterministically, by 'guessing' a retraction of each tree and an isomorphism between the resulting retracts. This gives the following theorem.

THEOREM 7.1. The word problem for any finitely generated free adequate semigroup or monoid is in $\mathcal{N P}$.

The exact computational complexity of the word problem remains unclear, and is deserving of further study. Notice that testing pruned trees for equivalence is exactly the isomorphism problem for labelled, rooted, retract-free, directed trees. Since such trees can be efficiently converted to expressions (via the map $\rho$ used in Section 5) it follows that the word problem for the free adequate monoid is at least as hard as this problem.

We now turn our attention to some structural properties of free adequate semigroups and monoids. Recall that the equivalence relation $\mathcal{J}$ is defined on any semigroup by $a \mathcal{J} b$ if and only if $a$ and $b$ generate the same principal two-sided ideal. A semigroup is called $\mathcal{J}$-trivial if no two elements generate the same principal two-sided ideal.

THEOREM 7.2. Every free adequate semigroup or monoid is $\mathcal{J}$-trivial.

Proof. Let $\Sigma$ be a set. By Theorems 5.16 and 5.17 it will suffice to show that $T^{1}(\Sigma)$ is $\mathcal{J}$-trivial.

Suppose that $X$ and $Y$ are pruned $\Sigma$-trees such that $X \mathcal{J} Y$ in $T^{1}(\Sigma)$. Then there exist $P, Q \in T^{1}(\Sigma)$ such that $Y=P X Q$. By Theorem $4.5, Y$ is isomorphic to $\overline{P \times X \times Q}$. By the definition of pruning there is a retraction $P \times X \times Q \rightarrow \overline{P \times X \times Q}$. Let $\sigma$ : $P \times X \times Q \rightarrow Y$ be the composition of this map with an isomorphism from $\overline{P \times X \times Q}$ to $Y$, and let $\gamma: X \rightarrow Y$ be the restriction of $\sigma$ to $X$, viewed as a subgraph of $P \times X \times Q$.

We claim first that the map $\gamma$ is in fact a morphism from $X$ to $Y$. Clearly, $\gamma$ preserves endpoints of edges; what remains is to show that it maps the start and end vertices of $X$ to the start and end vertices of $Y$, respectively. Since $\sigma$ is a morphism it clearly maps trunk edges of $P \times X \times Q$ injectively to trunk edges of $Y$. Since every trunk edge of $X$ is a trunk edge of $P \times X \times Q$ it follows that $\gamma$ maps trunk edges of $X$ injectively to trunk edges of $Y$. Thus, we conclude that $Y$ has at least as many trunk edges as $X$. But then by symmetry of assumption, $X$ and $Y$ have the same number of trunk edges, so $\gamma$ must map the trunk edges of $X$ bijectively onto the trunk edges of $Y$. It follows easily that $X$ maps the start and end vertices of $X$ to the start and end vertices of $Y$ respectively, as required to show that $\gamma$ is a morphism.

Now using symmetry of assumption again, we may also obtain a morphism $\delta: Y \rightarrow$ $X$. Consider now the composition $\delta \gamma: X \rightarrow X$. Since this is a map on a finite set, it has an idempotent power, say $(\delta \gamma)^{n}$, which is a retraction of $X$. Since $X$ is by assumption pruned, we conclude that $(\delta \gamma)^{n}$ is the identity map on $X$, and hence that $\gamma$ is injective on edges and vertices. In particular, we see that $Y$ has at least as many edges and 
vertices as $X$, and by symmetry of assumption once again, we may conclude that $X$ has the same number of edges and vertices as $Y$. It follows that the injective morphism $\gamma$ is surjective, which means that $\gamma$ is an isomorphism from $X$ to $Y$. Thus, $X$ and $Y$ represent the same element of $T^{1}(\Sigma)$.

THeorem 7.3. No free adequate semigroup or monoid on a nonempty set is finitely generated as a semigroup or monoid.

Proof. For each $X \in T^{1}(\Sigma)$, we let $\delta(X)$ be the greatest distance (length of an undirected path) of any vertex from the trunk. Clearly, $\delta(\bar{X}) \leq \delta(X)$ for all $X$. Moreover, for any pruned trees $X$ and $Y$ it follows easily from the definition of unpruned multiplication that $\delta(X \times Y)=\max (\delta(X), \delta(Y))$, so that

$$
\delta(X Y)=\delta(\overline{X \times Y}) \leq \delta(X \times Y)=\max (\delta(X), \delta(Y)) .
$$

Now if $F$ is any finite set of pruned $\Sigma$-trees, then there exists an upper bound on $\delta(X)$ for $X \in F$. It follows from the above that this is also an upper bound on $\delta(X)$ for $X$ in the subsemigroup of $T(\Sigma)$ (or the submonoid of $T^{1}(\Sigma)$ ) generated by $F$. But there are pruned trees in $T(\Sigma)$ with vertices arbitrarily far away from the trunk, so $F$ cannot generate the whole of these semigroups.

\section{Acknowledgements}

The author would like to thank J. B. Fountain and V. A. R. Gould for their encouragement and advice, all the authors of [1] for allowing him access to their unpublished work and work in progress, R. D. Gray for pointing out the connection between pruned trees and cores of graphs, and M. V. Lawson for alerting him to the existence of work on free restriction categories [2].

\section{References}

[1] M. J. J. Branco, G. M. S. Gomes and V. A. R. Gould, 'Left adequate and left Ehresmann monoids', Internat. J. Algebra Comput. 21(7) (2011), 1259-1284.

[2] J. R. B. Cockett and X. Guo, 'Stable meet semilattice fibrations and free restriction categories', Theory Appl. Categ. 16(15) (2006), 307-341 (electronic).

[3] J. R. B. Cockett and S. Lack, 'Restriction categories I. Categories of partial maps', Theoret. Comput. Sci. 270(1-2) (2002), 223-259.

[4] P. M. Cohn, Universal Algebra, 2nd edn, Mathematics and its Applications, Vol. 6 (D. Reidel, Dordrecht, 1981).

[5] J. Foniok, Homomorphisms and structural properties of relational system. PhD Thesis, Faculty of Mathematics and Physics, Charles University, Prague, 2007.

[6] J. B. Fountain, 'Adequate semigroups', Proc. Edinb. Math. Soc. (2) 22(2) (1979), 113-125.

[7] J. B. Fountain, 'Abundant semigroups', Proc. Lond. Math. Soc. 44 (1982), 103-129.

[8] J. B. Fountain, 'Free right $h$-adequate semigroups', in: Semigroups, Theory and Applications (Oberwolfach, 1986), Lecture Notes in Mathematics, Vol. 1320 (Springer, Berlin, 1988), pp. 97-120.

[9] J. B. Fountain, 'Free right type A semigroups', Glasgow Math. J. 33(2) (1991), 135-148. 
[10] J. B. Fountain, G. M. S. Gomes and V. A. R. Gould, 'The free ample monoid', Int. J. Algebra Comput. 19(4) (2009), 527-554.

[11] G. M. S. Gomes and V. A. R. Gould, 'Left adequate and left Ehresmann monoids II', J. Algebra 348(1) (2011), 171-195.

[12] V. A. R. Gould, '(Weakly) left E-ample semigroups'. Notes available online at www-users.york. ac.uk/ varg1/.

[13] C. Gutiérrez, 'On free inverse semigroups', Semigroup Forum 61(1) (2000), 154-158.

[14] F. Harary and M. D. Plummer, 'On the core of a graph', Proc. Lond. Math. Soc. (3) 17 (1967), 305-314.

[15] P. Hell and J. Nešetřil, 'The core of a graph', Discrete Math. 109(1-3) (1992), 117-126.

[16] M. Kambites, 'Retracts of trees and free left adequate semigroups', Proc. Edinb. Math. Soc. 54 (2011), 731-747.

[17] M. V. Lawson, 'Semigroups and ordered categories I: The reduced case', J. Algebra 141 (1991), 422-462.

[18] W. D. Munn, 'Free inverse semigroups', Proc. Lond. Math. Soc. (3) 29 (1974), 385-404.

[19] M. Petrich, 'Free inverse semigroups', Colloq. Math. 59(2) (1990), 213-221.

[20] O. Poliakova and B. M. Schein, 'A new construction for free inverse semigroups', J. Algebra 288(1) (2005), 20-58.

[21] M. A. Reynolds, 'A new construction for free inverse semigroups', Semigroup Forum 30(3) (1984), 291-296.

[22] H. E. Scheiblich, 'Free inverse semigroups', Semigroup Forum 4 (1972), 351-359.

[23] J. Stallings, 'Topology of a finite graph', Invent. Math. 71 (1983), 551-565.

\author{
MARK KAMBITES, School of Mathematics, University of Manchester, \\ Manchester M13 9PL, UK \\ e-mail: Mark.Kambites@manchester.ac.uk
}

